\title{
On voluntary ocular accommodation
}

\author{
ROBERT R. PROVINE \\ University of Maryland Baltimore County, 5401 Wilkins Avenue, Baltimore, Maryland 21228
}

and

JAY M. ENOCH

Department of Ophthalmology, University of Florida College of Medicine, Gainesville, Florida $\$ 2610$

\begin{abstract}
Young observers were challenged to induce a marked monocular accommodative response to a relatively weak accommodative stimulus by placing a-9 diopter contact lens on the eye. At first, observers could not produce the desired response, but with training, three of four subjects achieved criterion. Both a voluntary accommodative response and a response to an adequate accommodative stimulus were apparently involved. The voluntary component of the response could be demonstrated by having the observers repeat the task in total darkness.
\end{abstract}

Accommodation is a change in the curvature and thickness of the crystalline lens which is made in order to bring light from near objects into focus on the retina. At this time we have limited understanding of the nature of the adequate stimulus for accommodation (Campbell \& Westheimer, 1959) and the extent to which the response is innate (reflexive) or learned (Fincham, 1951; Heath, 1956; Campbell, 1959; Borish, 1970; Toates, 1972). It is therefore desirable to better understand the conditions under which accommodation may be induced.

In the present study, we examine the capacity of an observer to voluntarily induce positive accommodation in the absence of an adequate stimulus or in the presence of a markedly blurred stimulus. The blurred stimulus may approximate a nonstructured visual field (Whiteside, 1952; Westheimer, 1957; Fincham, 1962). We trained normal young observers to accommodate objects at infinity while wearing a -90 diopter (D) contact lens. This task had to be learned because observers had little prior experience with such a large step in accommodative demand in the absence of auxiliary distance cues and in the presence of a poor quality image. After the observers mastered this task, they were tested for their ability to induce accommodation in darkness in the absence of any visual stimulus.

\section{METHOD}

The subjects were four females between 16 and 18 years of age. All were enmetropic or manifested low hyperopia and had at least a $10 \mathrm{D}$ accommodative amplitude. The right eye was used in all experiments; the left eye was patched. Initially, the subjects viewed

The work on which this paper is based was conducted at the Washington University School of Medicine, St. Louis, Missouri. This work was supported in part by National Eye Institute Research Grant No. 7-R01-EY-01418-01. Reprint requests should be addressed to: Jay M. Enoch. Department of Ophthalmology, University of Florida College of Medicine, Gainesville, Florida 32610 . a monocularly presented stimulus pattern which consisted of two horizontal rectangles of light (each measuring $2^{\circ} 12^{\prime} \times 1^{\circ} 50^{\prime}$ and separated by $44^{\prime}$ measured at the entrance pupil of the eye). A small fixation point was centered between the two rectangles. The field was focused at intinity by the optical system, that is, the target array was located at the focal point of a field lens. The system was a classical Badal optometer (Ogle, 1961) incorporated in a Stiles-Crawford (S-C) apparatus (Enoch \& Hope, 1972) that was moditied for S-C peak finding determinations (Blank, Provine, \& Enoch, 1975). The S-C apparatus was used to present the target array. (Once the accommodative task described in this report was mastered, we sought to determine the effect of marked accommodation on photopic receptor orientation as estimated by the Stiles-Crawford effect.) The stimulus was red-orange (Wratten $23 \mathrm{~A}$ filter over a 6-V 15-A tungsten source), and the luminance level of the rectangles measured in the plane of the entrance pupil of the eye was $2.6 \log \mathrm{mL}$. The stimulus yielded a photopic response without producing glare. The two exit pupils of the instrument which were projected into the plane of the entrance pupil of the eye were $0.3 \mathrm{~mm}$ in diam. The two traces of the rectangles in the field stop were separated by $2 \mathrm{~mm}$ in the entrance pupil. These traces were polarized $90^{\circ}$ relative to each other. The fixation point was not polarized and therefore served as a Scheiner's disk target (e.g., Borish, 1970) aperture (two 0.3-mm-diam apertures separated by $2 \mathrm{~mm}$ ). Centuries ago, Father Scheiner devised a most sensitive systen for measuring when an object is in focus on the retina. He allowed light from a single object to pass two small apertures in the pupil. When the object in space is focused on the retina, the two light beams fuse to form a single image. Any other focal plane results in a double image with the beams either crossed or uncrossed.

The subject's eye and pupil were visualized by means of infrared (IR) light sources (tungsten bulbs with IR bandpass filters) and a RCA $6914 \mathrm{~A}$ infrared image converter unit which were part of the S-C test apparatus (Enoch \& Hope, 1972). The latter apparatus also had a bite bar and forehead press, which permitted the positioning of the observer's entrance pupil with $x, y, z$ controls.

For the experiment, subjects were fitted with a single $-9 \mathrm{D}$ soft (flexible, hydrophilic) contact lens (Bausch and Lomb, Inc., Sotlens) and placed in the Stiles-Crawford test device (Enoch \& Hope, 1972) which presented the previously described target at intinity (Blank. Provine, \& Enoch, 1975). Observers could focus the target by exerting an amount of positive accommodation equivalent to the negative power induced by the contact lens. Subjects were asked to accommodate on the target to the best of their ability for 4-6 sec and then to relax. Subsequent trials were begun after a $20-30-\mathrm{sec}$ rest period or when the observers signaled readiness. Subjects received feedback concerning their performance in two 
"lys: they could judge their own success in sharpening the test target and making the tixation spot single (Scheiner's disk-type stimulus. Borish, 1970), and in addition they received trial-by-trial comments from the experimenter. who estimated accommodative performance by observing the degree of the pupil contraction of the subject. ${ }^{1}$ Subjects pupils were visualized by means of the IR image converter. Atter each accommodative trial, the subjects were asked to estimate their performance on a $1-4$ graded scale, after which the experimenter commented on the degree of associated pupilary constriction.

\section{RESULTS AND DISCUSSION}

The stimulus array provided a relatively weak accommodative stimulus even when not blurred (few structural details in the field. near monochromatic light in a dark field, very small aperture dimensions). At first. none of the observers could accommodate on the target when a $-9 \mathrm{D}$ contact lens was placed on the eye. If a $-0.5 \mathrm{D}$ contact lens was substituted, no difficulty was encountered in clearing the target. Therefore. the variable involving the contact lens could be eliminated. Since the subjects had difficulty focusing the test stimulus while wearing the $-9 \mathrm{D}$ lens, we investigated the problem of inducing the desired acconmodative response.

As a first step in training accommodation. subjects were given experience in monocularly viewing objects held at about $10 \mathrm{~cm}$ from their right eyes. They were told to concentrate on what they experienced when they made the effort to focus upon such near objects. The subjects practiced the accommodation task daily between weekly experimental sessions. They were especially instructed to observe near objects, such as a pencil point held about $10 \mathrm{~cm}$ from the eye, and to notice how the background blurred when the pencil point was in focus. Subjects were then asked to attempt to blur the background in the same manner without the pencil point being in view.

These introductory training routines complemented test sessions using the Stiles-Crawford apparatus (Enoch \& Hope, 1972). When observers attempted to perform the assigned accommodative task in the apparatus, there was usually a great expenditure of effort. There was often an observable trembling of the head and body which necessitated continuous careful positioning of the eye by means of $x, y, z$ controls located on the Stiles-Craw ford apparatus. There was a tendency to stare during maximum accommodative effort. hence problems of lid effects on lens curvature were not significant. Lenses centered well. Brief periods of concentrated effort were used because of the considerable discomfort to observers and because lens drying tended to degrade image quality.

Observer performance during early trials was poor and erratic; entrance pupil contraction ${ }^{1}$ ranged from $0 \%$ to $20 \%$ of initial diameter (approximately $7-8 \mathrm{~mm}$ ) as estimated in reference to a precision reticule scale in the infrared image converter viewing system (the reticule was marked in $0.25-\mathrm{mm}$ steps). Only one of four subjects made progress toward mastering the task during the first test session. During the second test period a week later (the third week of the experiment). this subject was able to sharpen the target and frequently effected a $50 \%$ reduction in her pupil diameter ( $25 \%$ of initial pupil area). At this time. the subject became increasingly accurate in estimating her own performance at the task. There was a 1-3-sec latency between the time when an acconmodative effort $w$ as initiated and when maximum pupil constriction was noted. A similar course of events was followed by two of the three remaining subjects who eventually mastered the accommodation task during the next one to two test sessions. Once improvement in performance began, the observers rapidly learned the task. Subjects were considered to have mastered the task when they reported clearing the test target (a subjective criterion) and when they showed at least a $40 \%-60 \%$ reduction of pupil diameter (an externally observable criterion). It was difticult for the subjects to hold the tixation point single when they cleared the target (Scheiner's disk effect. e.g.. see Borish. 1970). When the fixation point was single, the target was properly focused on the retina. ${ }^{2}$

Experienced observers mentioned that the accommodation task required somewhat less effort after mastery was achieved. However, the task remained strenuous even after months of weekly $2-h$ experimental sessions. Subjects often reported "eye strain." headaches, dizziness, and in one case, nalusea. while performing the task, and these forms of discomfort occasionally persisted for several hours.

Evidence concerning the strategies used in solving the accommodation problem was obtained by means of oral and written reports provided by the subjects. Although all subjects reported that the key to the problem was found in concentrating on the task and in inagining they were looking at a near object. ${ }^{3}$ it is not clear why all subjects had low initial success rates, since this was reported as their consistent strategy from the beginning. Learning to "concentrate on." or "attend to," the difficult task was apparently an important factor. During early experimental sessions, any room noise or comment by the experimenter would seriously disrupt an ongoing effort. Experienced observers were less distractable. Observers' reports of attentional shifts or loss of concentration were often correlated with observed pupillary events. For example, a great deal of pupillary oscillation was often correlated with subjects' reports that they were unable to maintain attention.

Feedback appeared to play a varied, but important. role in learning the accommodative task. During early test trials, observers estimates of their own performance were poorly correlated with data on pupil sise. Verbal reports from inexperienced observers suggested that they equated their 
accommodative performance with the degree of physical effort expended on the task. This was evidently a poor criterion. However, during the period of rapid improvement in response, experimenter feedback was reported to be valuable. When subjects learned to induce sufficient accommodation aniplitude to clear the target, they had another more immediate and accurate source of feedback concerning their performance. The amount of blur would be reduced and the fixation point would become less double as the required accommodative response was approximated.

Three of the subjects who were able to reduce pupil diameter by approximately $40 \%-60 \%$ during accommodation would apparently induce more than $9 \mathrm{D}$ of accommodation in their initial response to the stimulus. This is suggested by their report that they overshot the endpoint in the test apparatus and had to come back to clear the stimulus. The experimenter often noted correlated pupillary oscillation in such cases, i.e., the pupil would constrict to some maximum value, then dilate slightly. The finding of such oscillation in the accommodative response suggests that while the induction and direction of the primary response were apparently voluntary, the establishment of the accommodative endpoint was mediated by stimulus properties. That the initial part of the response was most probably voluntary was evidenced by the finding that two experienced subjects who were asked to induce comparable accommodation in total darkness were able to reduce pupil diameter $40 \%-60 \%$, as estimated by the experimenter using the infrared viewer. This indicates that subjects can be trained to utilize internal performance criteria and are not dependent upon visual feedback for the initiation and/or short-term maintenance of an accommodative response. ${ }^{4}$

The observation that the subjects can reliably learn to accommodate for objects while wearing the $-9 \mathrm{D}$ lens further indicates that marked accommodation can be induced and maintained in situations which are freed from cues of apparent object distance. Although this fact has been known by clinicians and is used in their tests of positive and negative relative accommodation (e.g., Borish, 1970), these latter tests are not ordinarily performed using dioptic steps anywhere as great as the approximately $9 \mathrm{D}$ step used in this study.

Several investigators have previously demonstrated the presence of voluntary accommodation in a variety of tasks (Carr \& Allen, 1906; Sisson, 1938; Mark, 1962; Cornsweet \& Crane, 1973). However, none of the earlier studies attempted to produce as great an induced response as the one we describe. We showed that observers can be trained to induce nearly their entire accommodative amplitude. Furthermore, we were able to demonstrate the voluntary nature of the learned accommodative task by having observers produce it in total darkness.
Accommodation obviously involves a complex response mechanism. In this study, we posed a very difficult viewing task for the observers. On the basis of their performance, we infer the presence of a combined voluntary response coupled with a response to a relatively weak but adequate visual stimulus. We make this inference because the visual stimulus alone was not sufficient to induce the appropriate response. Therefore, some factor had to be learned and a similar response, once learned, could be elicited in total darkness on command. If this position is valid, then comparable but less extreme accommodative tasks may be learned in everyday experience. By extension, one may inquire as to the role of experience in the development of the initial accommodative response (Haynes, White, \& Held, 1965). Further, one may ask what effect the physical symptoms evidenced in attempts to accommodate have in specific clinical cases (Marg, 1951). This seems to be a most fertile ground for further investigative effort.

\section{REFERENCES}

Blank, K., Provine, R., \& Enoch, J. Shift in the peak of the photopic Stiles-Crawford effect with marked accomodation. Vision Research, 1975, in press.

Borish, I. Clinical refraction (3rd ed.). Chicago: Professional Press, 1970.

CAMPBELL, F. W. The accommodation response of the human eye. British Journal of Physiological Optics, 1959, 16, 188-203.

Campbell, F. W., \& Westheimer, G. Factors influencing accommodation responses of the human eye. Journal of the Optical Society of America, 1959, 49, 563-571.

CARR, H., \& AlleN, J. A study of certain relations of accommodation and convergence to the judgement of the third dimension. Psycholocial Review, 1906, 13, 258-275.

Cornsweet, T. N., \& CRANE, H. D. Training the visual accommodative system. Vision Research, 1973, 13, 713-715.

ENoch, J. M., \& Hope, G. M. An analysis of retinal receptor orientation. III. Results of initial psychophysical tests. Investigative Ophthalmology, 1972, 11, 765-782.

FINCHAM, E. F. The accommodation reflex and its stimulus. British Journal of Ophthalmology, 1951, 35, 381-393.

Fincham, E. F. Accommodation and convergence in the absence of retinal images. Vision Research, 1962, 1, 425-440.

HaYNes, H., White, B. L., \& Held, R. Visual accommodation in human infants. Science, 1965, 148, 428-530.

He ATH, G. Components of accommodation. American Journal of Optometry, 1956, 33, 569-579.

MARG, E. An investigation of voluntary as distinguished from reflex accommodation. American Journal of Optometry. 1951, 28, 347-356.

MARK, H. On the accuracy of accommodation. British Journal of Ophthalmology, 1962, 46, 742-744.

OGLE, K. Optics. Springfield, Illinois: Thomas, 1961. P. 235.

Sisson, E. D. Voluntary control of accommodation. Journal of General Psychology, 1938, 18. 195-198.

Toates, F. M. Accommodation function of the human eye. Physiological Review, 1972, 52, 828-871.

WESTHEIMER, G. Accommodation measurements in empty visual field. Journal of the Optical Society of America, 1957, 47, 714-718

WHITESIDE, T. Accommodation of the human eye in a bright and enipty visual field. Journal of Physiology (London), 1952. 118. $65 \mathrm{p}$. 


\section{NOTES}

1. It is well established that pupil contraction accompanies accommodation (e.g., Borish, 1970).

2. An observer's subjective reports of his ability to accommodate to the test stimulus has been objectively verified. In a separate exercise, a young male subject who was successful in inducing marked accommodation was retinoscoped during a trial external to the apparatus. He was found to be accommodating within 0.5 diopter of the experimental demand (Blank, Provine, \& Enoch, $1975)$ when requested to reproduce the experimental task $(-9 \mathrm{D}$ contact lens with intinity fixation).

3. In contrast, the two observers used by Cornsweet and Crane (1973) found that imagining an object moving toward or away from them was not helpful in learning the accommodative response.

4. It is perhaps relevant that Cornsweet and Crane (1973) have noted that there is a high degree of intertask transfer in voluntary accommodation tasks.

(Received for publication August 14, 1974; revision received October $31,1974$. 